\title{
Oxytocin Attenuates Amygdala Reactivity to Fear in Generalized Social Anxiety Disorder
}

\author{
Izelle Labuschagne ${ }^{1,6}, K_{\text {Luan Phan }}^{2,6}$, Amanda Wood ${ }^{3}$, Mike Angstadt' ${ }^{2}$, Phyllis Chua', Markus Heinrichs ${ }^{4}$, \\ Julie C Stout' and Pradeep J Nathan*,1,5,6 \\ 'School of Psychology and Psychiatry, Monash University, Melbourne, Australia; '2Department of Psychiatry, University of Michigan, Ann Arbor, \\ MI, USA; ${ }^{3}$ Department of Critical Care and Neurosciences, Murdoch Children's Research Institute, The Royal Children's Hospital, Melbourne, \\ Australia; ${ }^{4}$ Department of Psychology, University of Freiburg, Freiburg, Germany; ${ }^{5}$ Brain Mapping Unit, Department of Psychiatry, University of \\ Cambridge, Cambridge, UK
}

Patients with generalized social anxiety disorder (GSAD) exhibit heightened activation of the amygdala in response to social cues conveying threat (eg, fearful/angry faces). The neuropeptide oxytocin (OXT) decreases anxiety and stress, facilitates social encounters, and attenuates amygdala reactivity to threatening faces in healthy subjects. The goal of this study was to examine the effects of $O X T$ on fear-related amygdala reactivity in GSAD and matched healthy control (CON) subjects. In a functional magnetic resonance imaging study utilizing a double-blind placebo-controlled within-subjects design, we measured amygdala activation to an emotional face matching task of fearful, angry, and happy faces following acute intranasal administration of OXT ( $24 \mathrm{IU}$ or $40.32 \mu \mathrm{g}$ ) and placebo in I8 GSAD and I8 CON subjects. Both the CON and GSAD groups activated bilateral amygdala to all emotional faces during placebo, with the GSAD group exhibiting hyperactivity specifically to fearful faces in bilateral amygdala compared with the CON group. OXT had no effect on amygdala activity to emotional faces in the CON group, but attenuated the heightened amygdala reactivity to fearful faces in the GSAD group, such that the hyperactivity observed during the placebo session was no longer evident following OXT (ie, normalization). These findings suggest that OXT has a specific effect on fear-related amygdala activity, particularly when the amygdala is hyperactive, such as in GSAD, thereby providing a brain-based mechanism of the impact of OXT in modulating the exaggerated processing of social signals of threat in patients with pathological anxiety.

Neuropsychopharmacology (2010) 35, 2403-24I3; doi:I0.1038/npp.2010.123; published online I8 August 2010

Keywords: oxytocin; fear; emotion; amygdala; social anxiety disorder; functional magnetic resonance imaging (fMRI)

\section{INTRODUCTION}

Generalized social anxiety disorder (GSAD), also known as social phobia, is characterized by an exaggerated fear of negative scrutiny in several types of social situations. Notably, patients with GSAD have deficits in emotion processing, and specifically exhibit attentional bias for critical/aversive faces and threatening social signals (Amir et al, 2003; Clark and McManus, 2002; Foa et al, 2000; Mogg et al, 2004), and tend to avert their gaze from salient facial features such as the eyes (Bogels and Mansell, 2004; Garner et al, 2006; Horley et al, 2004) to reduce excessive anxiety during social interactions. These deficits have been strongly

*Correspondence: Professor PJ Nathan, Brain Mapping Unit, Department of Psychiatry, University of Cambridge, GSK Clinical Unit, Addenbroke's Hospital, Hills Road, Cambridge CB2 2GG, UK, Tel: + 44 I22 329 608I, Fax: + 44122329 6108,

E-mail: pn254@cam.ac.uk or izelle.labuschagne@med.monash.edu.au

${ }^{6}$ These authors contributed equally to this work.

Received 3 May 20 I0; revised 25 June 20 I0; accepted I 8 July 2010 linked to areas in the limbic system including the amygdala (Birbaumer et al, 1998; Blair et al, 2008; Etkin and Wager, 2007; Evans et al, 2008; Phan et al, 2006; Stein et al, 2002; Straube et al, 2004, 2005; Veit et al, 2002; Yoon et al, 2006), where threat-related hyperactivity is a cardinal pathophysiological feature in patients with GSAD (Etkin and Wager, 2007; Shin and Liberzon, 2010).

Several functional magnetic resonance imaging (fMRI) studies have shown that GSAD subjects exhibit heightened activation of the amygdala in response to social cues conveying threat (eg, fearful or angry faces) (Evans et al, 2008; Goldin et al, 2009; Phan et al, 2006; Stein et al, 2002; Straube et al, 2004). Selective-serotonin reuptake-inhibitors, often used to treat GSAD, can attenuate amygdala reactivity to fearful faces in healthy volunteers (Harmer et al, 2006) and in GSAD patients (Phan et al, unpublished data), and to public speaking in GSAD patients (Furmark et al, 2002), suggesting that the amygdala could serve as a brain target to test the underlying neural substrate of social behaviors and social anxiety. 
Extensive research in diverse species has shown that oxytocin (OXT), a neuropeptide produced in the hypothalamus of the brain, decreases anxiety and stress and facilitates social encounters (Bartz and Hollander, 2006) potentially by exerting an inhibitory effect via activation of GABAergic interneurons within the amygdala (Ehrlich et al, 2009; Huber et al, 2005). In humans, intranasal OXT has been shown to increase trust and willingness to accept social risks arising through interpersonal interactions (Kosfeld et $a l, 2005)$ and increase memory for positive social information (ie, happy faces; Rimmele et al, 2009), whereas suppressing physiological and subjective responses to stress (Heinrichs et al, 2003). Consistent with this evidence, functional imaging studies in healthy subjects have shown that equivalent doses of OXT attenuate amygdala reactivity to faces expressing fear and anger (Domes et al, 2007; Kirsch et al, 2005), as well as to fear-conditioned socially relevant faces (Petrovic et al, 2008) and in interpersonal interaction (Baumgartner et al, 2008), in the absence of overt changes in subjective mood or arousal (Domes et al, 2007; Kirsch et al, 2005; Petrovic et al, 2008). These findings are consistent with the distribution of oxytocin receptors (OXT-Rs) in areas associated with pro-social and anxiety behaviors such as the amygdala (Gimpl and Fahrenholz, 2001; Huber et al, 2005; Landgraf and Neumann, 2004; Vaccari et al, 1998; Yoshimura et al, 1993), and thus provide a putative brain-based mechanism to explore the underlying neural basis of OXT pharmacodynamic effects on the processing of social stimuli and related social behaviors, particularly as they relate to fear responding (Heinrichs et al, 2009).

The primary aim of this study was to use blood oxygenation level-dependent (BOLD) fMRI coupled with an emotional face matching task (EFMT) known to robustly and reliably activate the amygdala (Hariri et al, 2003) and which has been used in previous OXT studies in healthy controls (Kirsch et al, 2005), to examine the effects of OXT on amygdala reactivity to socially threatening faces in patients with GSAD and matched healthy control (CON) subjects. Given the evidence of heightened amygdala reactivity to social signals of threat (eg, fearful/angry faces) in GSAD (Phan et al, 2006; Stein et al, 2002), and of OXT's effects on amygdala response to aversive faces in previous fMRI studies (Domes et al, 2007; Kirsch et al, 2005), we employed an a priori region of interest (ROI) approach to test our primary hypothesis. Specifically, we hypothesized that OXT would (1) significantly attenuate threat related amygdala activity in both healthy controls and patients with GSAD, and (2) 'normalize' the threat-specific amygdala hyper-reactivity in GSAD subjects. In other words, we expected less amygdala reactivity to fearful/angry faces on OXT ( $v s$ placebo) in both groups, and the pattern of threatrelated hyper-activity observed at baseline (placebo) in GSAD ( $v s$ CON) subjects would no longer be evident following acute administration of OXT.

\section{MATERIALS AND METHODS}

\section{Subjects}

In all, 18 male GSAD subjects and 18 age- and gender-CON subjects, all right-handed and aged between 18-55 years, were included in the study and recruited via local newspaper and University advertisements (mean age \pm SD GSAD: $29.4 \pm 9.0$ years; age range: $20-55$ years) and CON: $29.9 \pm 10.2$ years; age range: $19-54$ years). Diagnosis of GSAD was established using the Clinical International Diagnostic Interview (CIDI Version 2.1; WHO, 1997) with additional probes from the Liebowitz Social Anxiety Scale (LSAS; Liebowitz, 1987), and verified by a physician interview. A score of $>70$ on the LSAS (including $>30$ on the 'social situations' subscale) were required to be included as the 'generalized' subtype. No GSAD subject had a current depressive episode (evident $\leqslant 6$ months) or alcohol/substance abuse (within 12 months of study entry), or another anxiety disorder (eg, generalized anxiety disorder, specific phobia, and panic disorder) that was more clinically salient or preceded GSAD as assessed using the CIDI, which utilizes DSMIV and ICD-10 criteria. The following comorbid disorders were evident, but clinically less salient than GSAD: agoraphobia (five subjects), conversion disorder (two subjects), specific phobia for nature/environment (one subject), panic disorder (one subject), pain disorder (one subject), hypochondriasis (one subject), and obsessive-compulsive disorder (one subject). Subjects were excluded if they had a history of post-traumatic stress disorder, bipolar disorder, psychotic disorder, mental retardation, or developmental disorders. The CON subjects had no history of a psychiatric disorder as verified by the CIDI. All subjects were non-smokers, free of head injury, had no allergies, and no history of alcohol or substance abuse. None of the subjects were on medication at the time of the study or were previously medicated. As part of the clinical screening, all subjects went through a brief medical examination with the study physician to assess that they were otherwise fit to take part in the study. Other clinical screening measures involved the Primary Care Evaluation of Mental Disorders (PRIME-MD; Spitzer et al, 1994), the Beck Depression Inventory (BDI-II; Beck et al, 1996), the Beck Anxiety Inventory (BAI; Beck and Steer, 1990), and two measures from the State-Trait Anxiety Inventory (STAI; Spielberg et al, 1983). After complete description of the study to the subjects, written informed consent was obtained. The study was conducted in accordance with the guidelines of the Standing Committee on Ethics in Research Involving Humans of Monash University.

\section{Study Design}

The study utilized a randomized, double-blind, placebocontrolled within-subject design, in which each participant was tested under two acute treatment conditions separated by a 1 week wash-out period. Treatment conditions were randomized and counterbalanced (by a clinical trials pharmacist, using a computerized randomization program in Windows Excel) with subjects receiving an intranasal spray of either OXT (24 IU or $40.32 \mu \mathrm{g}$; Syntocinon-spray; Novartis, Switzerland) or placebo (PBO, containing all ingredients except for the peptide) in three puffs of $4 \mathrm{IU}$ or $6.72 \mu \mathrm{g}$ per nostril. OXT or placebo was administered 45 min before fMRI scanning, consistent with previous studies (Domes et al, 2007; Heinrichs et al, 2003; Kirsch et al, 2005). Subjects arrived for each treatment session 
$\sim 1 \mathrm{~h}$ before the scheduled fMRI scanning session. No caffeine or alcohol intake was allowed on the day of scanning and no food permitted $1 \mathrm{~h}$ before arrival. Following a period of rest, subjects self-administered either an OXT or a placebo nasal spray as instructed by the investigator. This involved inhaling a full spray per nostril in an alternating order with 45-s wait between each application until total amount (ie, three sprays per nostril) was reached. The fMRI scanning started 45-min posttreatment (lasting $\sim 30 \mathrm{~min}$ ). This time window was chosen to coincide with the time of oxytocin's predicted maximum pharmacokinetic (Born et al, 2002) and physiological effects (Domes et al, 2007; Heinrichs et al, 2003; Kirsch et al, 2005).

\section{EFMT}

The subjects were assessed using an EFMT (Hariri et al, 2002), which has been previously used in our pharmacological fMRI studies (Phan et al, 2008). This task involved photographs from a validated set of face stimuli (Gur et al, 2002), which has shown to reliably and robustly activate the amygdala and its use has been effective in other pharmacological fMRI studies (Hariri et al, 2000; Kirsch et al, 2005; Paulus et al, 2005; Tessitore et al, 2002). Using a blockdesign, participants viewed a trio of faces and selected one of two faces (bottom) that expressed the same emotion as the target face (top). The identity of all three faces was always different, and an equal number of male and female faces were presented. The target and congruent probe faces displayed one of three expressions (fearful, angry, or happy), and the other (incongruent) probe face always displayed a neutral/nonemotional expression. This design allowed us to isolate amygdala reactivity specifically to each emotional expression, which have similar perceptual characteristics except for the threat/nonthreat signal conveyed. To maintain attention and allow limbic (ie, amygdala) brain responses to return to baseline, the face matching tasks were interspersed with a 'baseline' task, in which subjects matched simple geometric shapes (circles, rectangles, or triangles) similar to instructions above. The paradigm consisted of 18 experimental blocks, each containing four sequential matching trials of 3-s presentation (ie, 12-s blocks). The 18 blocks consisted of 9 blocks of matching emotional faces ( 3 blocks of each target expression of fearful, angry, and happy), interleaved with 9 blocks of matching shapes, counterbalanced across two runs. Participants responded to the tasks by pressing the left or right response buttons with their right hand. The task was presented visually on a computer using Presentation software (http://www.neurobehavioralsystems.com) and deflected onto a screen. Participants' response accuracy and reaction times were analyzed in a 2 group (GSAD, $\mathrm{CON}) \times 2$ drug $(\mathrm{OXT}, \mathrm{PBO}) \times 3$ emotion (fearful, angry, happy) repeated-measures ANOVA, Greenhouse-Geisser corrected where necessary. Significant main effects and interactions were followed by post hoc paired $t$-tests to examine the effect of OXT.

\section{fMRI Acquisition and Statistical Analysis}

Brain images were acquired with a 3T Siemens Tim Trio scanner using a 12-channel head coil. Functional gradient-echo echo-planar imaging (EPI) data depicting BOLD contrast were acquired during the experimental task $(\mathrm{TE}=40 \mathrm{~ms}$, $\mathrm{TR}=3000 \mathrm{~ms}, \quad$ flip-angle $=90^{\circ}, \quad \mathrm{FoV}=210 \mathrm{~mm}, \quad 64 \times 64$ matrix, 44 contiguous $3 \mathrm{~mm}$ slices parallel to the hippocampus, and interleaved). Whole brain T1-weighted anatomical reference images were also acquired from all participants $\left(\mathrm{TE}=2.15 \mathrm{~ms}, \mathrm{TR}=1900 \mathrm{~ms}\right.$, flip-angle $=9^{\circ}, \mathrm{FoV}=$ $256 \mathrm{~mm}, 176$ sagittal slices, $1 \mathrm{~mm}$ slice thickness, perpendicular to the AC-PC line). Data processing and analyses were performed using Statistical Parametric Mapping (SPM5) software. Data from 2 participants (1 CON and 1 GSAD subject) was discarded because of poor data quality, resulting in a final analysis of 17 GSAD and $17 \mathrm{CON}$ subjects. Images were spatially realigned to correct for head motion, warped to an EPI template in Montreal Neurological Institute space, and resampled to $2 \mathrm{~mm}^{3}$ kernel. The general linear model was applied to the time series, convolved with the canonical hemodynamic response function (Friston et al, 1995) with a 128-s high-pass filter. Condition effects were modeled with box-car regressors representing the occurrence of each block type, and effects were estimated at each voxel, and for each subject before group analyses. Individual contrast maps (Statistical Parametric Maps) were then analyzed at the second level in a random effects statistical model (Holmes and Friston, 1998) for within-group, between-group, and between-session analyses.

Although we had a priori hypotheses about oxytocin's effects within the amygdala in response to threat (angry and/or fearful) faces, to obviate bias and generate exploratory findings for subsequent hypotheses, we conducted a whole-brain voxel-wise analysis of variance (ANOVA) with drug (OXT, PBO) and emotion (fear, angry, happy $v s$ shapes) as within-subject factors, and group (GSAD, CON) as between-subject factor. The significance threshold was set at $p<0.001$ (uncorrected) with a cluster extend threshold of $k=10$. We were particularly interested in the main and/or interaction effects of OXT, and thus explicitly examined main effect of drug and drug-related interactions (drug $\times$ group, drug $\times$ emotion and group $\times \operatorname{drug} \times$ emotion) across the entire brain.

To test our a priori hypotheses, we also took a ROI analysis approach selectively for amygdala activity. Briefly, because the primary hypothesis was highly specific to amygdala and its response to threat ( $v s$ nonthreat/happy) faces, and to the direction of OXT effects (ie, attenuation), and was related to the often observed differences in amygdala reactivity in GSAD, we chose to approach the ROI analysis in a way that would minimize selection bias (Kriegeskorte et al, 2009) using a functional ROI localizer that is not particularly dependent on any one specific factor (threat, OXT, GSAD) (Poldrack, 2007; Poldrack and Mumford, 2009); this 'functional localizer' is the best representation of the activations exhibited by the cohort of subjects studied. Here, similar to numerous pharmacofMRI studies that have used this task (Arce et al, 2008; Hariri et al, 2002; Kirsch et al, 2005; Paulus et al, 2005; Phan et al, 2008), we initially measured an overall amygdala response to the EFMT using the linear contrast estimate of BOLD signal difference between all faces (fearful, angry, and happy, combined) vs shapes thereby obviating bias toward one particular emotion expression; in addition to avoid bias 
toward a particular diagnosis or drug condition, we averaged amygdala response (all faces $>$ shapes) across all subjects (GSAD, CON) and across scan session (OXT, PBO), totaling 68 individual contrast images. Focal activation of the amygdala was tested for significance at a stringent whole-brain voxel-wise threshold of $p<0.05$ family-wise error corrected for multiple comparisons across the entire brain. On the basis of strong a priori evidence across studies and from our own laboratory (Adolphs, 2003; Costafreda et al, 2008; Fusar-Poli et al, 2009; Phan et al, 2002, 2008; Zald, 2002), we expected robust bilateral amygdala activation to these emotional faces, compared with the nonsocial control 'shapes'. From these activation clusters, we created a functional ROI for the left and right amygdala composed of a $10 \mathrm{~mm}$ radius sphere surround the peak activation (all faces $>$ shapes), representing 515 total voxels and a volume of $4120 \mathrm{~mm}^{3}$ on each side. We then used these functional ROIs to extract BOLD signal responses (parameter estimates, $\beta$-weights in arbitrary units) of activation averaged across all 515 voxels (for left and right amygdala separately) from each GSAD and CON subject for each emotional condition separately (fearful faces $>$ shapes, angry faces $>$ shapes, happy faces $>$ shapes) and from each session separately (OXT, PBO). We chose to use a relatively large $10 \mathrm{~mm}$ radius spherical ROI to maximize coverage and be inclusive of the functional activations of the 34 subjects in our sample, who show inter-individual variability in the exact location of the activation in the amygdala in response to the EFMT, as also demonstrated by two recent metaanalyses (Costafreda et al, 2008; Fusar-Poli et al, 2009); previous papers have utilized similar approaches and volumes for the amygdala ROI (Blasi et al, 2009; Williams et al, 2006). The functional ROI extractions were then used to test our a priori hypotheses by comparing the extent of left and right amygdala reactivity to each emotion by: (1) using a repeated measures ANOVA with emotion (fearful, angry, and happy) and drug (OXT, PBO) as within-subject factors, and group (GSAD, CON) as between-subject factor; and (2) using post hoc $t$-tests for follow-up analyses for (a) between GSAD and CON subjects during PBO and during OXT sessions separately, using independent samples $t$-tests; and (b) between PBO and OXT sessions in GSAD and CON groups separately, using paired $t$-tests. For these planned $t$-test comparisons, significance was set at $p<0.05$, two-tailed.

\section{Subjective Mood and Statistical Analysis}

Changes in subjective mood was assessed twice, once upon arrival (and before drug administration) and once following drug administration (succeeding the scan session) using the Visual Analogue Mood Scale (VAMS; Bond and Lader, 1974). The VAMS consists of 16 bipolar items composed of two adjectives with opposite feelings, which are presented on a $100 \mathrm{~mm}$ line. These items were combined into three main factors to include: (1) alertness, (2) contentedness, and (3) calmness. The VAMS data were analyzed to examine change in scores over time following drug administration (post- minus pre-treatment), and were determined within each group and drug condition in a 2 group (GSAD, $\mathrm{CON}) \times 2$ drug $(\mathrm{OXT}, \mathrm{PBO}) \times 3$ factor (alertness, contentedness, calmness) repeated-measures ANOVA.

\section{RESULTS}

\section{Demographic and Clinical Characteristics}

The characteristics of the groups are reported in Table 1. The CON and GSAD groups were matched on age and education with no significant difference between the groups on these variables. The GSAD group scored significantly higher on all clinical measures including the LSAS, BDI-II, BAI, and STAI. Of note, the GSAD subjects scored (mean \pm SD) $43.56 \pm 8.6$ and $38.11 \pm 11.0$ on the social situations and performance subscales, respectively, of the LSAS (total score: $81.67 \pm 17.5$ ), suggesting broad and moderate-severe impairment.

\section{Behavioral Performance (EFMT) During Scanning}

Button-press response data from one GSAD subject was not successfully recorded because of technical failure, therefore, giving a final sample size of 17 for the GSAD group. Overall task performance was very high, with subjects making on average $92.6 \%( \pm \mathrm{SEM}=0.8)$ correct responses during the entire paradigm. OXT relative to $\mathrm{PBO}$ did not affect emotion recognition or response times in either of the GSAD or CON groups. Figure 1 presents the means and standard deviations for accuracy (ie, percentage correct) and reaction times (milliseconds) for the individual emotions (fear, angry, and happy). For accuracy, a repeated-measures ANOVA revealed that there were no significant performance differences for group $(F(1,66)=2.19, p=0.144)$ or drug $(F(1,66)=0.618, p=0.434)$ and no significant interactions among group, drug, or emotion (group $\times$ drug: $F(1,66)=0.005, \quad p=0.942$; group $\times$ emotion: $F(2,132)=$ $0.330, p=0.720$; drug $\times$ emotion: $F(2,132)=0.224, p=0.800$; group $\times$ drug $\times$ emotion: $F(2,132)=0.238, p=0.789)$. A significant main effect was evident for emotion $(F(2,132)=28.22$, $p<0.0005)$ with highest-to-lowest accuracy in the following pattern: happy $>$ fear $>$ angry (percentage mean \pm SEM: $97.63 \pm 1.0,94.33 \pm 1.1,86.28 \pm 1.9$, respectively) across groups and drug conditions. For reaction times, repeated-measures

Table I Demographic and Clinical Characteristics

\begin{tabular}{|c|c|c|c|c|}
\hline & CON & GSAD & $t_{(34)}^{a}$ & $p$ \\
\hline Age & $29.89(10.2)$ & $29.39(9.0)$ & 0.156 & 0.877 \\
\hline Education & $16.00(2.5)$ & $14.67(1.6)$ & 1.930 & 0.062 \\
\hline BDI-II & $1.28(1.9)$ & $10.83(7.5)$ & -5.265 & $<0.001$ \\
\hline BAl & $2.17(5.0)$ & I $6.89(8.2)$ & -6.503 & $<0.001$ \\
\hline State anxiety (STAI) & $23.59(7.0)$ & $38.8 \mid(15.8)$ & -6.135 & $<0.001$ \\
\hline Trait anxiety $(\mathrm{STAl})^{\mathrm{b}}$ & $27.35(8.2)$ & $50.44(11.5)$ & -7.785 & $<0.001$ \\
\hline LSAS: total & I3.94 (8.3) & $81.67(17.5)$ & -14.828 & $<0.001$ \\
\hline LSAS: performance & $6.89(5.1)$ & $38.11(11.0)$ & -10.916 & $<0.001$ \\
\hline LSAS: social situations & $7.06(4.6)$ & $43.56(8.6)$ & -15.876 & $<0.001$ \\
\hline
\end{tabular}

Abbreviations: CON, healthy control subjects; GSAD, generalized social anxiety disorder subjects; BDI-II, Beck Depression Inventory Version II; BAI, Beck

Anxiety Inventory; STAI, State-Trait Anxiety Inventory; LSAS, Liebowitz Social Anxiety Scale.

Data are expressed as mean $( \pm S D) ; n=18$ per group.

annaired $t$-tests, two-tailed.

${ }^{b}$ Data missing from two GSAD $(n=16)$ and one CON $(n=17)$ subjects. 

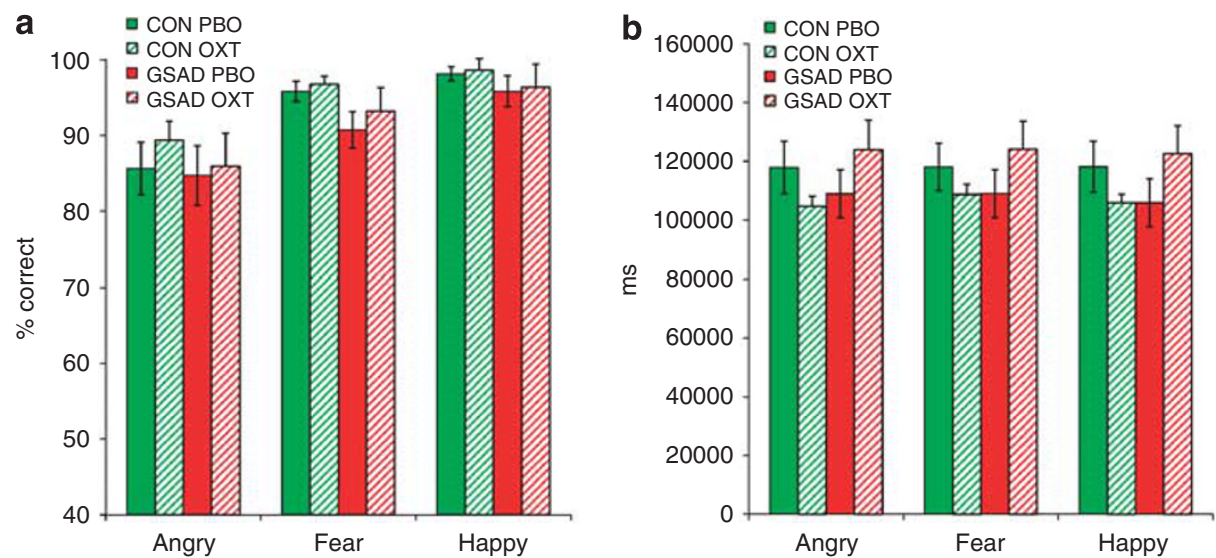

Figure I (a) Accuracy (percentage correct) and (b) reaction times (milliseconds) in the emotional face matching task following placebo/PBO and oxytocin/OXT treatment. Data are expressed as mean ( \pm SEM).

ANOVA revealed that there were no difference in response times across all main effects (group: $F(1,66)=0.211, p=0.647$; drug: $F(1,66)=0.067, p=0.796$; emotion: $F(2,132)=2.489$, $p=0.106$ ), as well as no significant interactions (group $\times$ drug: $F(1,66)=3.164, p=0.080$; group $\times$ emotion: $F(2,132)=1.990$, $p=0.192$; drug $\times$ emotion: $F(2,132)=0.715, p=0.491$; groupdrug $\times$ emotion: $F(2,132)=1.013, p=0.366)$.

\section{Functional MRI Data}

Whole-brain analyses. Whole-brain findings for the main and interaction effects of drug are summarized in Table 2. The whole-brain voxel-wise analysis did not detect significant main effects of drug, or interactions related to drug (drug $\times$ emotion, drug $\times$ group, drug $\times$ emotion $\times$ group) within the amygdala.

ROI Analyses. As expected, across all subjects and drug conditions, we observed robust bilateral amygdala activation to emotional (fearful, angry, and happy) faces, compared with the nonsocial control shapes: (right amygdala: $(20,-4,-22)$, Z-score $=6.08, \quad p_{\text {corrected }}<0.0005$, volume $=904 \mathrm{~mm}^{3}$; left amygdala: $(-18,-6,-20), Z$-score $=7.28, p_{\text {corrected }}<0.0005$, volume $=1272 \mathrm{~mm}^{3}$; Figure 2). Analysis of extracted BOLD signal from the bilateral amygdala spherical ROIs functionally derived from these activation peaks (Figure 3 ) revealed that both groups activated (positive $\beta$-weights) amygdala bilaterally in response to fearful, angry, and happy faces separately during PBO treatment (Table 3); this confirms the expected response to the EFMT (Adolphs, 2003; Costafreda et al, 2008; Fusar-Poli et al, 2009; Phan et al, 2002, 2008; Zald, 2002) and allows for inferences about oxytocin's effects on amygdala activation. Table 3 shows the magnitude ( $\beta$-weights \pm SEM) of amygdala activation within each group from the OXT and PBO sessions separately. When we entered extracted BOLD signal ( $\beta$-weights) from the functional amygdala ROIs into repeated-measures ANOVA for left and right amygdala separately, we observed a trend level significance for emotion $\times$ drug $\times$ group interaction for left and right amygdala $(F(2,64)=2.79, p=0.069$, and $F(2,64)=2.21, p=0.118$, respectively). The main effects of emotion, drug and group were non-significant $\left(p_{s}>0.2\right)$. Subsequent post hoc t-test analyses revealed that the three-way interaction was driven by a hyperactivity and OXT effects in the patient group. That is, as predicted, GSAD subjects had greater amygdala reactivity to fearful faces in both left and right amygdala compared with CON subjects during PBO treatment (Figure 4). Following OXT treatment, the exaggerated amygdala response to fearful faces in GSAD subjects ( $>\mathrm{CON}$ ) observed during the PBO session was no longer evident (Figure 4). As expected, in GSAD subjects, amygdala reactivity to fearful faces was greater during $\mathrm{PBO}$ than during OXT treatment, showing that OXT reduced fear-related amygdala activation (Figure 4). Unlike GSAD subjects, control participants did not exhibit differences in amygdala activation to fearful faces between OXT and PBO treatments (Figure 4). No between-group differences (GSAD vs CON) in the extent of amygdala activation were observed for angry and happy faces on OXT or PBO treatment (Table 3). Moreover, no between-session (OXT vs $\mathrm{PBO}$ ) differences in the extent of amygdala activation were observed for angry and happy in either the GSAD or $\mathrm{CON}$ group (Table 3). As a final analysis, we tested for drugorder effects and found no drug-order effects on amygdala activation ( $p$ 's $>0.05)$.

\section{Subjective Mood}

There were no effects of OXT (relative to $\mathrm{PBO}$ ) on the VAMS factors in both the CON and GSAD group (means and SD are presented in Table 4). The repeated-measures ANOVA did not reveal significant main effects for group $(F(1,68)=0.283, p=0.596)$ or drug $(F(1,68)=0.147$, $p=0.703)$ or interactions for group $\times \operatorname{drug}(F(1,68)=0.145$, $p=0.705)$, drug $\times$ factor $(F(2,136)=1.281, \quad p=0.281) \quad$ or group $\times$ drug $\times$ factor $(F(2,136)=0.084, p=0.878)$. A significant interaction was evident for group $\times$ factor $(F(2,136)=$ 7.078, $p=0.003$ ), such that regardless of drug administration, the GSAD patients showed significantly greater mood changes post-treatment compared with that of the CON group; patients showing significantly more calmness (mean \pm SEM: $\mathrm{CON}=-1.04 \pm 1.7, \mathrm{GSAD}=7.56 \pm 2.3, p=0.026$ ), and less alertness $(\mathrm{CON}=-1.74 \pm 1.8, \mathrm{GSAD}=-6.88 \pm 2.0, p=0.061)$ following drug administration, and no group difference was evident for contentedness $(\mathrm{CON}=-0.66 \pm 1.3$, GSAD $=$ $-0.81 \pm 1.3, p=0.424)$. 
Table 2 Whole-Brain Voxel-Wise ANOVA for Main Effect of Drug and Drug-Related Interactions on the EFMT ${ }^{\mathrm{a}}$

\begin{tabular}{|c|c|c|c|c|c|c|c|c|}
\hline \multirow[t]{2}{*}{ Region } & \multirow[t]{2}{*}{ Side } & \multicolumn{3}{|c|}{ Coordinates } & \multirow{2}{*}{$\begin{array}{c}\text { Cluster } \\
k\end{array}$} & \multirow{2}{*}{$\begin{array}{l}\text { Volume } \\
V\left(\mathrm{~mm}^{3}\right)\end{array}$} & \multicolumn{2}{|c|}{ Voxel } \\
\hline & & $x$ & $y$ & $z$ & & & $\mathbf{F}$ & $z$ \\
\hline \multicolumn{9}{|l|}{ Drug } \\
\hline Brainstem & L & -4 & -20 & -48 & 55 & 440 & $|4.2|$ & 3.5 \\
\hline Calcarine fissure & $\mathrm{R}$ & 20 & -64 & 8 & 24 & 192 & 14.25 & 3.51 \\
\hline Caudate & $L$ & -14 & -10 & 22 & 11 & 88 & 14.72 & 3.57 \\
\hline Cerebellum & L & -20 & -36 & -44 & 13 & 104 & 14.65 & 3.56 \\
\hline \multirow[t]{2}{*}{ Cingulate, middle } & $\mathrm{R}$ & 18 & -36 & 46 & 816 & 6528 & 26.33 & 4.79 \\
\hline & L & -18 & -38 & 46 & 359 & 2872 & 23.4 & 4.52 \\
\hline Cuneus & L & 0 & -92 & 36 & 18 & 144 & 14.45 & 3.54 \\
\hline Lingual & $\mathrm{R}$ & 6 & -62 & -2 & 34 & 272 & 14.17 & 3.5 \\
\hline Occipital, inferior & $\mathrm{R}$ & 40 & -94 & -6 & 26 & 208 & 16.53 & 3.79 \\
\hline Occipital, middle & L & -24 & -72 & 0 & 127 & 1016 & 23.87 & 4.57 \\
\hline Occipital, superior & L & -18 & -100 & 16 & 46 & 368 & 17.11 & 3.86 \\
\hline \multirow[t]{2}{*}{ Parahippocampal } & $R$ & 36 & -34 & -14 & 52 & 416 & 18.05 & 3.97 \\
\hline & L & -12 & -12 & -24 & 53 & 424 & 22.77 & 4.46 \\
\hline Parietal, inferior & L & -24 & -76 & 44 & 10 & 80 & 12.42 & 3.26 \\
\hline \multirow[t]{2}{*}{ Postcentral } & $\mathrm{R}$ & 44 & -18 & 30 & 27 & 216 & 16 & 3.73 \\
\hline & L & -34 & -24 & 40 & 10 & 80 & 12.64 & 3.29 \\
\hline Precentral & L & -28 & -10 & 42 & 87 & 696 & 19.33 & 4.11 \\
\hline \multirow[t]{2}{*}{ Supramarginal } & $\mathrm{R}$ & 64 & -24 & 28 & 269 & 2152 & 16.17 & 3.75 \\
\hline & L & -60 & -38 & 30 & 46 & 368 & 14.18 & 3.5 \\
\hline Temporal, middle & L & -66 & -30 & 8 & 21 & 168 & 15.32 & 3.64 \\
\hline \multirow[t]{2}{*}{ Temporal, superior } & $\mathrm{R}$ & 62 & -30 & 10 & 23 & 184 & 15.37 & 3.65 \\
\hline & L & -40 & 0 & -16 & 34 & 272 & | 4.65 & 3.56 \\
\hline Thalamus & L & -8 & -20 & 10 & 20 & 160 & 13.88 & 3.46 \\
\hline
\end{tabular}

\begin{tabular}{|c|c|c|c|c|c|c|}
\hline \multicolumn{7}{|l|}{ Drug $\times$ group } \\
\hline Brainstem & L & -14 & $-20-40$ & 42 & 336 & | 7.55 3.91 \\
\hline Caudate & L & -6 & $18 \quad-8$ & 21 & 168 & 14.923 .6 \\
\hline \multirow[t]{2}{*}{ Frontal, inferior } & $\mathrm{R}$ & 64 & 26 & 33 & 264 & 21.074 .29 \\
\hline & L & -38 & 22 & 11 & 88 & $|4.4| 3.53$ \\
\hline Frontal, superior & L & -20 & $22-14$ & 38 & 304 & 17.193 .87 \\
\hline Fusiform & L & -24 & $-82-12$ & 14 & 112 & 14.883 .59 \\
\hline Occipital, middle & $L$ & -30 & -92 & 60 & 480 & | 4.923 .59 \\
\hline
\end{tabular}

$\begin{array}{lllllllll}\text { Drug } \times \text { emotion } & & & & & & & \\ \quad \text { Fusiform } & \text { R } & 38 & -18 & -28 & 20 & 160 & 8.5 & 3.42 \\ \text { Temporal, middle } & \text { R } & 52 & -52 & -2 & 20 & 160 & 8.5 & 3.42\end{array}$

Drug $\times$ group $\times$ emotion

Cerebellum

$\llcorner\quad-14 \quad-58-32$

83

664

10.993 .98

aSignificance threshold at $p<0.00$ I (uncorrected) with a cluster extent threshold of $k$ (number of contiguous voxels) $\geqslant 10$.

\section{DISCUSSION}

Modulation of the amygdala has been suggested to have a critical role in oxytocin's effects on pro-social- and anxietyrelated behavior. Patients with GSAD show amygdala hyperactivity to threatening social cues and we reasoned

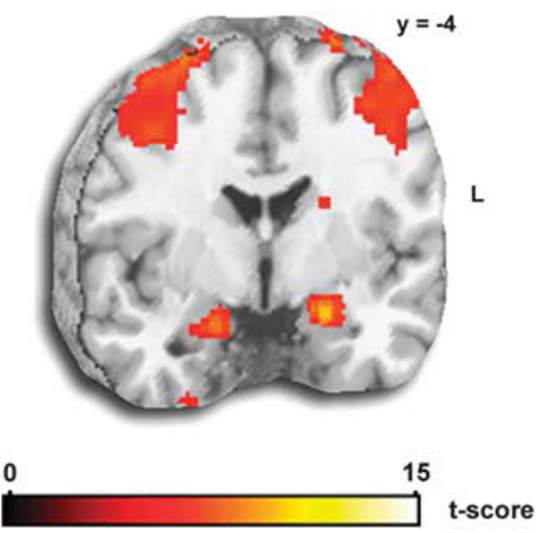

Figure 2 Task-related activation (all faces > shapes) of bilateral amygdala. Statistical t-map overlaid on a canonical brain rendering (MNI coronal $y$-plane $=-4$ ) showing greater left and right amygdala reactivity to emotional faces (vs shapes) from all subjects (GSAD and CON) across both OXT and PBO sessions. Whole brain voxel-wise statistical map showing all significant activations at the threshold of $p<0.05$, family-wise error corrected for multiple voxel-wise comparisons across the entire brain. Activation details described in Results.

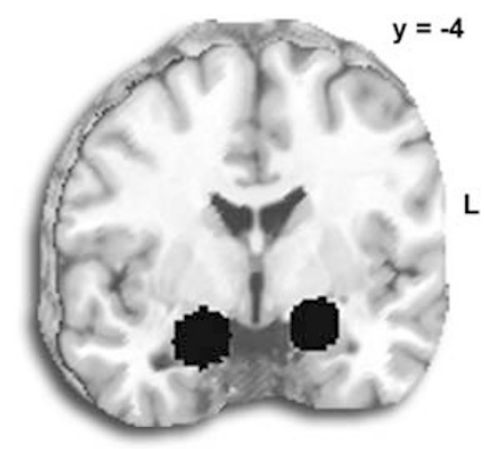

Figure 3 A two-dimensional view of the spherical $\mathrm{ROI}$ ( $10 \mathrm{~mm}$ radius) of the left and right amygdala, from which the parameter estimates were extracted.

that if OXT can attenuate this hyperactivity, then it may provide a neural mechanism to facilitate processing of social stimuli and related social behaviors including social threat. Supporting our hypotheses, we observed that patients with GSAD showed bilateral amygdala hyperactivity specifically to fearful faces, relative to controls. OXT had no effect on amygdala activity to emotional faces in healthy controls, but attenuated the heightened amygdala reactivity to fearful faces in patients with GSAD, such that the hyperactivity observed in patients during the placebo session was no longer evident following OXT (ie, normalization).

In patients with GSAD, the amygdala hyperactivity was observed for fearful faces, but not happy faces, supporting previous suggestions that the hyperactivity is specific to aversive threatening social cues (Phan et al, 2006). The fearrelated hyperactivity is consistent with a number of previous studies in GSAD where exaggerated amygdala reactivity has been reported to aversive social cues including fearful faces (Phan et al, 2006) and to 'harsh' (fearful, angry, and contemptuous) faces (Phan et al, 2006; 
Table 3 Within- and Between-Group Comparison of Extracted BOLD Signal ( $\beta$-Weights) from the Functional Amygdala ROls (Left and Right) in Response to Each Emotional (Fearful, Angry, and Happy) Face from Each Group (GSAD, CON) for Each Imaging and Treatment Session (OXT, PBO)

\begin{tabular}{|c|c|c|c|c|c|c|c|c|c|c|c|c|}
\hline & & & \multirow{2}{*}{\multicolumn{2}{|c|}{ Paired $t$-test }} & \multirow{2}{*}{\multicolumn{2}{|c|}{ GSAD }} & & & \multicolumn{4}{|c|}{ Group differences } \\
\hline & \multicolumn{2}{|c|}{ CON } & & & & & \multicolumn{2}{|c|}{ Paired $t$-test } & \multicolumn{2}{|c|}{ PBO t-test } & \multicolumn{2}{|c|}{ OXT t-test } \\
\hline \multicolumn{13}{|l|}{ Left } \\
\hline Angry & $0.174(0.06)$ & $0.136(0.05)$ & 0.606 & 0.55 & $0.034(0.07)$ & $0.121(0.05)$ & 1.035 & 0.32 & 1.484 & 0.15 & 0.22 & 0.83 \\
\hline Happy & $0.147(0.06)$ & $0.161(0.03)$ & 0.253 & 0.8 & $0.137(0.04)$ & $0.084(0.04)$ & 0.867 & 0.4 & 0.121 & 0.9 & 1.475 & 0.15 \\
\hline \multicolumn{13}{|l|}{ Right } \\
\hline Angry & $0.169(0.05)$ & $0.111(0.07)$ & 0.862 & 0.4 & $0.121(0.07)$ & $0.176(0.04)$ & 0.653 & 0.52 & 0.549 & 0.59 & 0.816 & 0.42 \\
\hline Fearful & $0.076(0.05)$ & $0.05 \mid(0.05)$ & 0.538 & 0.6 & $0.297(0.08)$ & $0.137(0.06)$ & 1.865* & 0.08 & $2.393 * *$ & 0.02 & 1.184 & 0.25 \\
\hline
\end{tabular}

Abbreviations: CON, healthy control subjects; GSAD, generalized social anxiety disorder subjects; OXT, oxytocin; PBO, placebo.

Data are expressed as mean $( \pm$ SEM); $n=17$ per group. $* p<0.05$, one-tailed; $* * * 0.05$, two-tailed.

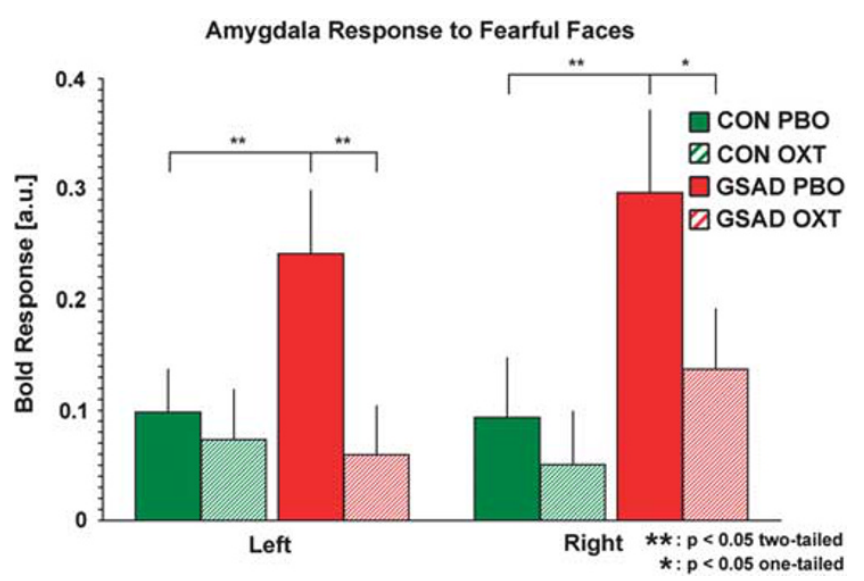

Figure 4 Extracted BOLD responses (mean $\beta$-weights, arbitrary units (a.u.)) to fearful faces extracted from the left and right amygdala within each group for each session, showing: (I) greater amygdala reactivity in GSAD group (>CON) during placebo/PBO treatment; (2) attenuation of amygdala reactivity in GSAD group by oxytocin/OXT treatment (PBO > OXT); and (3) extent of amygdala reactivity is similar in GSAD and CON groups on OXT. Asterisks denote between-group and betweensession differences, ${ }^{*} * p<0.05$, two-tailed; $* p<0.05$, one-tailed).

Stein et al, 2002). However, we did not observe similar amygdala hyperactivity to angry faces, which has previously been reported in a number of studies (Evans et al, 2008; Phan et al, 2006; Stein et al, 2002; Straube et al, 2004). While we expected amygdala hyperactivity to 'harsh' (angry and fearful) faces, as each convey social signals of threat, the lack of hyperactivity to angry faces and the apparent inconsistency may be explained by a number of factors. For example, previous studies have shown amygdala activations to angry facial expressions are less intense compared with fearful faces (Fitzgerald et al, 2006; Whalen et al, 2001), whereas others have shown no amygdala activation to angry faces (Blair et al, 1999; Fusar-Poli et al, 2009), suggesting that activation to angry faces may not only be less intense,
Table 4 Behavioral Changes in Visual Analogue Mood Scale (VAMS) Factors Pre- and Post Treatment (PBO, OXT) in Both Participant Groups (CON, GSAD)

\begin{tabular}{|c|c|c|c|c|c|}
\hline \multirow[t]{2}{*}{ Factor } & \multirow[t]{2}{*}{ Time } & \multicolumn{2}{|c|}{ CON } & \multicolumn{2}{|c|}{ GSAD } \\
\hline & & PBO & OXT & PBO & OXT \\
\hline \multirow[t]{2}{*}{ Alertness } & Pretreatment & $69.3(3.0)$ & $66.9(3.3)$ & $51.5(3.1)$ & $53.9(2.9)$ \\
\hline & Post-treatment & $68.1(2.7)$ & $64.6(3.8)$ & $46.0(3.6)$ & $45.6(3.0)$ \\
\hline \multirow[t]{2}{*}{ Contentedness } & Pretreatment & $76.3(2.1)$ & $75.5(2.0)$ & $56.9(3.0)$ & $56.4(2.8)$ \\
\hline & Post-treatment & $74.6(2.6)$ & $75.8(2.0)$ & $57.8(2.7)$ & $57.0(2.7)$ \\
\hline \multirow[t]{2}{*}{ Calmness } & Pretreatment & $69.1(2.9)$ & $71.0(3.0)$ & $55.6(3.4)$ & $50.2(3.5)$ \\
\hline & Post-treatment & $68.6(3.7)$ & $73.6(2.3)$ & $61.7(2.6)$ & $59.3(3.2)$ \\
\hline
\end{tabular}

Abbreviations: CON, healthy control subjects; GSAD, generalized social anxiety disorder subjects; OXT, oxytocin; PBO, placebo.

Data are expressed as mean ( \pm SEM); $n=18$ per group.

but also more variable. The latter findings may be a consequence of the relative differences in the recognition of fearful $v s$ angry faces. Our behavioral findings showed that angry faces were overall less accurately recognized compared with fearful and happy expressions across groups, consistent with similar findings by others in healthy controls and patients with social phobia (Guastella et al, 2009; Joormann and Gotlib, 2006). Psychological models of emotional processing also suggest a more complex and divergent processing of facial cues conveying anger relative to fear. Anger, although commonly related to aversive contexts, is viewed as an approach-related behavior as it involves approaching particular desired outcomes, which may involve the creation of discomfort for someone else or of rectifying an injustice (Carver and Harmon-Jones, 2009). Hence, angry faces may have been viewed with some ambiguity resulting in both reduced amygdala activity, as well as behavioral face recognition accuracy. 
In healthy human subjects, OXT has been shown to attenuate amygdala response to perception of threatening or aversive faces (ie, angry and fearful faces; Domes et al, 2007; Kirsch et al, 2005), as well as fear-conditioned socially relevant faces (Petrovic et al, 2008). Our findings advance this existing literature by showing for the first time that OXT can also attenuate clinically abnormal fear-related hyperactivity of the amygdala in patients with GSAD, such that the extent of fear response was not different to that in healthy controls ('normalization'). Notably, the effects of OXT was specific to the fear-related hyperactivity observed in patients with GSAD, as OXT did not attenuate the amygdala activity to fear in the healthy control subjects, as well as the amygdala activity to angry and happy faces in both groups. However, these findings need to be interpreted with caution as these findings are in direct contrast to previous studies in healthy subjects described above, where OXT was shown to attenuate amygdala response to angry and fearful faces (Domes et al, 2007; Kirsch et al, 2005), as well as have a general effect on amygdala suppression regardless of valence (Domes et al, 2007). We provide the following interpretations to synthesize discrepant findings. First, it is possible that the effects of OXT on amygdala response to faces in healthy subjects are more variable than originally thought. The two previous fMRI studies in healthy subjects (Domes et al, 2007; Kirsch et al, 2005) reported small effects in the amygdala and the inconsistency of findings across studies, including the current investigation, may be due to the small sample sizes (13 subjects in both Domes et al, and Kirsch et al, and 17 controls in ours). Second, our EFMT task consisted of only three blocks per emotion, and therefore, the analysis of amygdala signal, particular in the healthy subjects who may have less sensitivity to these emotional faces, may be underpowered to detect OXT effects. Our null funding (in the largest fMRI study on OXT to date) and a recent study showing enhanced amygdala response to fearful faces in healthy women (Domes et al, 2010), suggests that at least in healthy subjects, the effects of OXT on amygdala response to facial cues may be variable and/or more subtle. Moreover, effects of OXT may also be moderated by individual differences in trait variables (eg, trait anxiety). A recent study has also shown that OXT may have differential effects on the activity of specific amygdala subregions during processing of emotional faces (Gamer et al, 2010). Future studies are needed to clarify the role of these important factors as they relate to oxytocin's effects on brain function in healthy humans.

The specific effects of OXT on fear in patients with GSAD also suggest an intriguing possibility that OXT may have the greatest and most consistent effects on amygdala activity when the amygdala is dysfunctional such as the hyperactivity observed in patients with GSAD. Under these conditions, OXT may be able to reduce activity to 'normal' levels of function. Therefore, from an evolutionary prospective, OXT's role could be to specifically modulate amygdala under pathological rather than normal physiological states (eg, to reduce the processing of threatening social cues after the initial threat has been detected), as it would be undesirable to have a general dampening of the amygdala to all emotional cues. Such a mechanism could also explain why OXT specifically attenuated the amygdala response to fear rather than a general suppression of response to all faces regardless of valence. Alternatively, the apparent specificity for fearful faces may be due to OXT having differential effects on processing of fear relative to anger and happy faces. Indeed, OXT has shown improved recognition memory for angry faces (Savaskan et al, 2008) and increased encoding, and subsequent retrieval of happy faces (relative to threatening faces; Guastella et al, 2008), suggesting that consolidation of these faces in memory (via amygdala-hippocampal interactions) may explain the lack of attenuation of angry- and happy-related amygdala activity following OXT. However, the apparent fear specific attenuation of amygdala activity by OXT in GSAD and our explanations of the findings require confirmation and warrant further investigation.

The effect of OXT in normalizing fear-related amygdala hyperactivity in patients with GSAD suggests that the amygdala may be critical target for OXT's pharmacodynamic effects on the processing of social stimuli and related social behaviors. In support, increased release of OXT from local OXTergic fibers has been noted in the central nucleus of the amygdala during stressful or anxietyprovoking situations (De Vries and Buijs, 1983; Landgraf and Neumann, 2004), and behavioral changes in fear and anxiety correlate with differences in the levels of OXT-R expression and the potency of OXT-R antagonism (Bale et al, 2001; Champagne and Meaney, 2001; Ebner et al, 2005; Landgraf and Neumann, 2004; Lubin et al, 2003). Furthermore, there is evidence that a sub-population of GABAergic interneurons in the amygdala are activated by OXT-R stimulation (Huber et al, 2005). These GABAergic interneurons are part of the intra-amygdala system of inhibitory GABAergic connections, which are thought to integrate the output activity of the central nucleus of the amygdala (Cassell et al, 1999; Savander et al, 1996). OXT's physiological effects may indeed be mediated by the inhibition of amygdala output pathways that are critical for the behavioral and physiological expression of fear.

OXT's effect on fear-related amygdala activity was observed in the absence of overt subjective changes in mood or anxiety. These findings are entirely consistent with previous neuroimaging (Kirsch et al, 2005) and behavioral (Di Simplicio et al, 2008; Fischer-Shofty et al, 2010; Rimmele et al, 2009; Unkelbach et al, 2008) and clinical (Guastella et al, 2009) studies, which have shown similar insensitivity of behavioral and clinical (ie, LSAS) measures of mood and anxiety following single doses or short-term administration of OXT. OXT also failed to affect behavioral task performance (ie, accuracy and reaction times for face matching) consistent with previous studies that have shown no effects of OXT on an identical face matching task (Kirsch et $a l, 2005)$ and face recognition using facial cues of similar intensity (ie, 100\%; Di Simplicio et al, 2008; Marsh et al, 2010). However, OXT has been shown to enhance speed of recognition of moderately intense fear (Di Simplicio et al, 2008) and happy faces (Marsh et al, 2010), as well as dynamic fearful faces (Fischer-Shofty et al, 2010), suggesting that behavioral effects of OXT may be greater when requiring greater interpersonal processing. These data suggest that fMRI may be more sensitive in detecting changes in neural processing that lead up to social behavioral changes. Hence, it is possible that by modulating amygdala response to threat-related cues, prolonged treatment 
with OXT may reveal clinical and behavioral improvements in patients with GSAD, as OXT in humans appears to enhance the ability to interact socially (Kosfeld et al, 2005) and improve control over stress and anxiety in social interactions (Heinrichs et al, 2003). Recent studies, have also shown a relationship between social anxiety symptoms and plasma OXT levels in GSAD patients (Hoge et al, 2008), and that administering OXT improves patients' self-evaluation of appearance and speech performance, albeit without additive effect on exposure treatment outcomes (Guastella et al, 2009). The current acute findings provide a proof of mechanism in support of further studies to examine the potential anxiolytic and pro-social effects of chronic OXT or OXT-R agonists in patients with GSAD. Future studies are needed to clarify the differential effects, if any, between acute and more chronic administration of OXT on behavior, anxiety symptomatology, and brain function.

Several limitations of the current study should be noted. First, our whole-brain analysis did not yield a significant drug $\times$ group $\times$ emotion interaction for the amygdala. This may have been due to the small sample studied and there was insufficient power to test this three-way interaction. Second, our main contrast of interest used the shapes condition as the 'control', to maximize amygdala activation (Hariri et al, 2000); moreover, because the neutral face stimuli was present within each trial of face trio, we could not isolate out the neutral faces as a separate condition. Therefore, we cannot ascertain if these findings would hold had we been able to use neutral faces as an alternate control condition. Also, given the lack on an additional 'control'/ baseline condition (eg, fixation/rest), we cannot determine group or drug effects in brain response to the matching shapes condition. Third, it is possible that our laboratory/ fMRI study environment (ie, entering the laboratory and going through MRI scanning) may have impacted on subjective mood and this may have potentially confounded any drug effects. However, our behavioral findings on mood are consistent with previous OXT studies where similar assessments and procedures were performed. Finally, our findings in males cannot be extended to females, particularly as gender differences could have a role in modulating the neural effects of OXT (Domes et al, 2010), as well as the amygdala reactivity to emotional faces (Killgore and Yurgelun-Todd, 2001; Lee et al, 2002). Future studies are needed to address these important issues.

In conclusion, data from this study showed that OXT has a specific effect on fear-related amygdala activity, particularly, when the amygdala is hyperactive, such as in GSAD. By showing that OXT can 'normalize' this hyperactivity in socially anxious individuals who exhibit aberrant fear responses, the current data provides initial evidence for a brain-based mechanism involving OXT-Rs within the amygdala, previously posited to involve inhibitory effects via GABAergic interneurons, thereby, suppressing the behavioral and physiological expression of fear and facilitating social behavior.

\section{ACKNOWLEDGEMENTS}

This work was supported by a NARSAD Independent Investigator Award and a grant from Monash University to
Pradeep J Nathan. K Luan Phan and Mike Angstadt were supported by NIH Grant MH076198. Costs for oxytocin nasal sprays were supported by a Grant from the Swiss National Science Foundation (SNSF PP001-114788) to Markus Heinrichs. Amanda Wood was supported by an Australian Rotary Health fellowship.

\section{DISCLOSURE}

The authors IL, KLP, AW, MA, PC, MH, JS, and PJN declare that no financial support or compensation has been received from any individual or corporate entity over the past 3 years for research or professional service and there are no personal financial holdings that could be perceived as constituting a potential conflict of interest. PJN is also an employee at GlaxoSmithKline Pharmaceuticals, but has no conflict of interest with this study, which was performed at Monash University where he holds an academic appointment.

\section{REFERENCES}

Adolphs R (2003). Is the human amygdala specialized for processing social information. Ann N Y Acad Sci 985: 326-340.

Amir N, Klumpp H, Przeworski A (2003). Attentional bias to threat in social phobia: facilitated processing of threat or difficulty disengaging attention from threat? Behavr Res Ther 41: 1325-1335.

Arce E, Simmons AN, Lovero KL, Stein KL, Paulus MP (2008). Escitalopram effects on insula and amygdala BOLD activation during emotional processing. Psychopharmacology (Berl) 196: 661-672.

Bale TL, Davis AM, Auger AP, Dorsa DM, McCarthy MM (2001). CNS Region-specific oxytocin receptor expression: importance in regulation of anxiety and sex behavior. J Neurosci 21: 2546-2552.

Bartz JA, Hollander E (2006). The neuroscience of affiliation: forging links between basic and clinical research on neuropeptides and social behaviour. Harm Behav 50: 518-528.

Baumgartner T, Heinrichs M, Vonlanthen A, Fischbacher U, Fehr E (2008). Oxytocin shapes the neural circuitry of trust and trust adaptation in humans. Neuron 58: 639-650.

Beck A, Steer R (1990). Manual for the Beck Anxiety Inventory. Psychological Corporation: San Antonio, TX.

Beck A, Steer R, Brown G (1996). Manual for Beck Depression Inventory-II. Psychological Corporation: San Antonio, Tex.

Birbaumer N, Grodd W, Diedrich O, Klose U, Erb M, Lotze M et al (1998). fMRI reveals amygdala activation to human faces in social phobias. Neuroreport 9: 1223-1226.

Blair K, Geraci M, Devido J, McCaffrey D, Chen G, Vythilingam M et al (2008). Neural response to self- and other referential praise and criticism in generalized social phobia. Arch Gen Psychiatry 65: 1176-1184.

Blair RJR, Morris JS, Frith CD, Perret DI, Dolan RJ (1999). Dissociable neural responses to facial expressions of sadness and anger. Brain 122: 883-893.

Blasi G, Popolizio T, Taurisano P, Caforio G, Romano R, Di Giorgio A et al (2009). Changes in prefrontal and amygdala activity during olanzapine treatment in schizophrenia. Psychiatr Res 173: 31-38.

Bogels SM, Mansell W (2004). Attention processes in the maintenance and treatment of social phobia: hypervigilance, avoidance and self-focused attention. Clin Psychol Rev 24: 827-856. 
Bond AJ, Lader MH (1974). The use of analogue scales in rating subjective feelings. Br J Med Psychol 47: 211-218.

Born J, Lange T, Kern W, McGregor GP, Bickel U, Fehm HL (2002). Sniffing neuropeptides: a transnasal approach to the human brain. Nat neurosci 5: 514-516.

Carver CS, Harmon-Jones E (2009). Anger is an approach-related affect: evidence and implications. Psychol Bull 135: 183-204.

Cassell MD, Freedman LJ, Shi c (1999). The intrinsic organization of the central extended amygdala. Ann N Y Acad Sci 877: 217-241.

Champagne F, Meaney MJ (2001). Naturally occurring variations in maternal behavior in the rate are associated with differences in estrogen-inducible central oxytocin receptors. Proc Natl Acad Sci USA 98: 12736-12741.

Clark DM, McManus F (2002). Information processing in social phobia. Biol Psychiatry 51: 92-100.

Costafreda SG, Brammer MJ, David AS, Fu CH (2008). Predictors of amygdala activation during the processing of emotional stimuli: a meta-analysis of 385 PET and fMRI studies. Brain Res Rev 58: 57-70.

De Vries GJ, Buijs RM (1983). The origin of the vasopressinergic and oxytocinergic innervation of the rat brain with special reference to the lateral septum. Brain Res 273: 307-317.

Di Simplicio M, Massey-Chase R, Cowen PJ, Harmer CJ (2008). Oxytocin enhances processing of positive versus negative emotional information in healthy male volunteers. J Psychopharmacol 23: 241-248.

Domes G, Heinrichs M, Gläscher J, Büchel C, Braus DF, Herpertz SC (2007). Oxytocin attenuates amygdala responses to emotional faces regardless of valence. Biol Psychiatry 62: 1187-1190.

Domes G, Lischke A, Berger C, Grossmann A, Hauenstein K, Heinrichs $\mathrm{M}$ et al (2010). Effects of intranasal oxytocin on emotional face processing in women. Psychoneuroendocrinology 35: 83-93.

Ebner K, Bosch OJ, Kromer SA, Singewald N, Neumann ID (2005). Release of oxytocin in the rat central amygdala modulates stresscoping behavior and the release of excitatory amino acids. Neuropsychopharmacology 30: 223-230.

Ehrlich I, Humeau Y, Grenier F, Ciocchi S, Herry C, Luthi A (2009). Amygdala inhibitory circuits and the control of fear memory. Neuron 62: 757-771.

Etkin A, Wager TD (2007). Functional neuroimaging of anxiety: a meta-analysis of emotional processing in PTSD, social anxiety disorder, and specific phobia. Am J Psychiatry 164: 1476-1488.

Evans KC, Wright CI, Weding MM, Gold AL, Pollack MH, Rauch SL (2008). A functional MRI study of amygdala responses to angry schematic faces in social anxiety disorder. Depression Anxiety 25: 496-505.

Fischer-Shofty M, Shamay-Tsoory SG, Hariri AR, Levkovitz Y (2010). The effect of intranasal administration of oxytocin on fear recognition. Neuropsychologia 48: 179-184.

Fitzgerald DA, Angstadt M, Jelsone LM, Nathan PJ, Phan KL (2006). Beyond threat: amygdala reactivity across multiple expressions of facial affect. Neuroimage 30: 1441-1448.

Foa EB, Gilboa-Schechtman E, Amir N, Freshman M (2000). Memory bias in generalized social phobia: remembering negative emotional expressions. J Anxiety Disord 14: 501-519.

Friston KJ, Holmes AP, Worsley KJ, Poline JB, Frith CD, Frackowiak RS (1995). Statistical parametric maps in functional imaging: a general linear approach. Hum Brain Mapp 2: 189-210.

Furmark T, Tillfors M, Marteinsdottir I, Fischer H, Pissiota A, Langstrom B et al (2002). Common changes in cerebral blood flow in patients with social phobia treated with citalopram or cognitivebehavioral therapy. Arch Gen Psychiatry 59(5): 425-433.

Fusar-Poli P, Placentino A, Carletti F, Landi P, Allen P, Surguladze $S$ et al (2009). Functional atlas of emotional face processing: a voxel-based meta-analysis of 105 functional magnetic resonance imaging studies. J Psychiatry Neurosci 34: 418-432.
Gamer M, Zurowski B, Büchel C (2010). Different amygdala subregions mediate valence-related and attentional effects of oxytocin in humans. Proc Natl Acad Sci USA 107: 9400-9405.

Garner M, Mogg K, Bradley BP (2006). Fear-relevant selective associations and social anxiety: absence of a positive bias. Behav Res Ther 44: 201-217.

Gimpl G, Fahrenholz F (2001). The oxytocin receptor system: structure, function, and regulation. Physiol Rev 81: 629-683.

Goldin PR, Manber T, Hakimi S, Canli T, Gross JJ (2009). Neural bases of social anxiety disorder: emotional reactivity and cognitive regulation during social and physical threat. Arch Gen Psychiatry 66: 170-180.

Guastella AJ, Carson DS, Dadds MR, Mitchell PB, Cox RE (2009). Does oxytocin influence the early detection of angry and happy faces? Psychoneuroendocrinology 34: 220-225.

Guastella AJ, Mitchell PB, Mathews F (2008). Oxytocin enhances the encoding of positive social memories in humans. Biol Psychiatry 64: 256-258.

Gur RC, Sara R, Hagendoorn M, Marom O, Hughett P, Macy L et al (2002). A method for obtaining 3-dimensional facial expressions and its standardization for use in neurocognitive studies. J Neurosci Meth 115: 137-143.

Hariri AR, Bookheimer SY, Mazziotta JC (2000). Modulating emotional responses: effects of a neocortical network on the limbic system. Neuroreport 11: 43-48.

Hariri AR, Mattay VS, Tessitore A, Fera F, Weinberger DR (2003). Neocortical modulation of the amygdala response to fearful stimuli. Biol Psychiatry 53: 494-501.

Hariri AR, Tessitore A, Mattay VS, Fera F, Weinberger DR (2002). The amygdala response to emotional stimuli: a comparison of faces and scenes. Neuroimage 17: 317-323.

Harmer CJ, Mackay CE, Reid CB, Cowen PJ, Goodwin GM (2006). Antidepressant drug treatment modifies the neural processing of nonconscious threat cues. Biol Psychiatry 59(9): 816-820.

Heinrichs M, Baumgartner T, Kirschbaum C, Ehlert U (2003). Social support and oxytocin interact to suppress cortisol and subjective responses to psychosocial stress. Biol Psychiatry 54 1389-1398.

Heinrichs M, von Dawans B, Domes G (2009). Oxytocin, vasopressin, and human social behavior. Front Neuroendocrinol 30: $548-557$.

Hoge EA, Pollack MH, Kaufman RE, Zak PJ, Simon NM (2008). Oxytocin levels in social anxiety disorder. CNS Neurosci Thera 14: $165-170$

Holmes AP, Friston KJ (1998). Generalisability, random effects and population inference. Neuroimage 7: S754.

Horley K, Williams LM, Gonsalvez C, Gordon E (2004). Face to face: visual scanpath evidence for abnormal processing of facial expressions in social phobia. Psychiatry Res 127: 43-53.

Huber D, Veinante P, Stoop R (2005). Vasopressin and oxytocin excite distinct neuronal populations in the central amygdala. Science 308: 245-248.

Joormann J, Gotlib IH (2006). Is this happiness I see? Biases in the indentification of emotional facial expressions in depression and social phobia. J Abnorm Psychol 115: 705-714.

Killgore WD, Yurgelun-Todd DA (2001). Sex differences in amygdala activation during the perception of facial affect Neuroreport 12: 2543-2547.

Kirsch P, Esslinger C, Chen Q, Mier D, Lis S, Siddhanti S et al (2005). Oxytocin modulates neural circuitry for social cognition and fear in humans. J Neurosci 25: 11489-11493.

Kosfeld M, Heinrichs M, Zak PJ, Fischbacher U, Fehr E (2005). Oxytocin increases trust in humans. Nature 435: 673-676.

Kriegeskorte N, Simmons WK, Bellgowan PSF, Baker CI (2009). Circular analysis in systems neuroscience: the dangers of double dipping. Nat neurosci 12: 535-540.

Landgraf R, Neumann ID (2004). Vasopressin and oxytocin release within the brain: a dynamic concept of multiple and variable 
modes of neuropeptide communication. Front Neuroendocrinol 25: $150-176$.

Lee TM, Liu HL, Hoosain R, Liao WT, Wu CT, Yuen KS et al (2002). Gender differences in neural correlates of recognition of happy and sad faces in humans assessed by functional magnetic resonance imaging. Neurosci Lett 333: 13-16.

Liebowitz MR (1987). Social Phobia. Mod Probl Pharmacopsychiatry 22: 141-173.

Lubin DA, Elliot JC, Black MC, Johns JM (2003). An oxytocin antagonist infused into the central nucleus of the amygdala increases maternal aggressive behavior. Behav Neurosci 117: 195-201.

Marsh AA, Yu HH, Pine DS, Blair RJ (2010). Oxytocin improves specific recognition of positive facial expressions. Psychopharmacology (Berl) 209: 225-232.

Mogg K, Philippot P, Bradley BP (2004). Selective attention to angry faces in clinical social phobia. J Abnorm Psychol 113: 160-165.

Paulus MP, Feinstein JS, Castillo G, Simmons AN, Stein MB (2005). Dose-dependent decrease of activation in bilateral amygdala and insula by lorazepam during emotion processing. Arch Gen Psychiatry 62: 282-288.

Petrovic P, Kalisch R, Singer T, Dolan RJ (2008). Oxytocin attenuates affective evaluations of conditioned faces and amygdala activity. J Neurosci 28: 6607-6615.

Phan KL, Angstadt M, Golden J, Onyewuenyi I, Popovska A, de Wit $\mathrm{H}$ (2008). Cannabinoid modulation of amygdala reactivity to social signals of threat in humans. J Neurosci 28: 2313-2319.

Phan KL, Fitzgerald DA, Nathan PJ, Tancer ME (2006). Association between amygdala hyperactivity to harsh faces and severity of social anxiety in generalised social phobia. Biol Psychiatry 59: 424-429.

Phan KL, Wager TD, Taylor SF, Liberzon I (2002). Functional neuroanatomy of emotion: a meta-analysis of emotion activation studies in PET and fMRI. Neuroimage 16: 331-348.

Poldrack RA (2007). Region of interest analysis for fMRI. Social Cogn Affect Neurosci 2: 67-70.

Poldrack RA, Mumford JA (2009). Independence in ROI analysis: where is the voodoo? SCAN 4: 208-213.

Rimmele U, Hediger K, Heinrichs M, Klaver P (2009). Oxytocin makes a face in memory familiar. J Neurosc 29: 38-42.

Savander V, Go CG, LeDoux JE, Pitkanen A (1996). Intrinsic connections of the rat amygdaloid complex: projections originating in the accessory basal nucleus. J Comp Neurol 37: 291-313.

Savaskan E, Ehrhardt R, Schulz A, Walter M, Schächinger H (2008). Post-learning intranasal oxytocin modulates human memory for facial identity. Psychoneuroendocrinology 33: 368-374.

Shin LM, Liberzon I (2010). The neurocircuitry of fear, stress, and anxiety disorders. Neuropsychopharmacology 35: 169-191.
Spielberg CD, Gorsuch RL, Lushene RE (1983). Manual for the State-Trait Anxiety Inventory.. Consulting Psychologist Press: Palo Alto, CA.

Spitzer RL, Williams JB, Kroenke K, Linzer M, DeGruy III FV, Hahn SR et al (1994). Utility of a new procedure for diagnosing mental disorders in primary care. The PRIME-MD 1000 study. JAMA 14: 1749-1756.

Stein MB, Goldin PR, Sareen J, Zorrilla LTE, Brown GG (2002). Increased amygdala activation to angry and contemptuous faces in generalized social phobia. Arch Gen Psychiatry 59: 1027-1034.

Straube T, Kolassa I, Glauer M, Mentzel H, Miltner WHR (2004). Effect of task conditions on brain responses to threatening faces in social phobics: an event-related functional magnetic resonance imaging study. Biol Psychiatry 56: 921-930.

Straube T, Mentzel H, Miltner WHR (2005). Common and distinct brain activation to threat and safety signals in social phobia. Neuropsychobiology 52: 163-168.

Tessitore A, Hariri AR, Fera F, Smith WG, Chase TN, Hyde TM et al (2002). Dopamine modulates the response of the human amygdala: a study in Parkinson's disease. J Neurosci 22: 9099-9103.

Unkelbach C, Guastella AJ, Forgas JP (2008). Oxytocin selectively facilitates recognition of positive sex and relationship words. 19: 1092-1094.

Vaccari C, Lolait SJ, Ostrowski NL (1998). Comparative distribution of vasopressin $\mathrm{V} 1 \mathrm{~b}$ and oxytocin receptor messenger ribonucleic acids in brain. Endocrinology 139: 5015-5033.

Veit R, Flor H, Erb M, Hermann C, Lotze M, Grodd W et al (2002). Brain circuits involved in emotional learning in antisocial behaviour and social phobia in humans. Neurosci Lett 328: 233-236.

Whalen PJ, Shin LM, McInerney SC, Fischer H, Wright CI, Rauch SL (2001). A functional MRI study of human amygdala responses to facial expressions of fear versus anger. Emotion 1: 70-83.

WHO, World Health Organization Composite International Diagnostic Interview (CIDI, Version 2.1, 1997 World Health Organization: Geneva.

Williams LM, Das P, Liddell BJ, Kemp AH, Rennie CJ, Gordon E (2006). Mode of functional connectivity in amygdala pathways dissociates level of awareness for signals of fear. J Neurosci 26: 9264-9271.

Yoon KL, Fitzgerald DA, Angstadt M, McCarron A, Phan KL (2006). Amygdala reactivity to emotional faces at high and low intensity in generalised social phobia: a 4-Tesla functional fMRI study. Psychiatry Res Neuroimaging 154: 93-98.

Yoshimura R, Kiyama H, Kimura T, Araki T, Maeno H, Tanizawa $\mathrm{O}$ et al (1993). Localisation of oxytocin receptor messenger ribonucleic acid in the rat brain. Endocrinology 133: 1239-1246.

Zald DH (2002). The human amygdala and the emotional evaluation of sensory stimuli. Brain Res Rev 41: 88-123. 